\title{
Gérer les aléas. Les sociétés du Haut Atlas marocain
}

Martine Garrigues-Cresswell et Béatrice Lecestre-Rollier

\section{(2) OpenEdition}

Journals

Édition électronique

URL : https://journals.openedition.org/tc/230

DOI : $10.4000 /$ tc. 230

ISSN : 1952-420X

Éditeur

Éditions de l'EHESS

Édition imprimée

Date de publication : 1 mars 2002

ISSN : 0248-6016

Référence électronique

Martine Garrigues-Cresswell et Béatrice Lecestre-Rollier, « Gérer les aléas. Les sociétés du Haut Atlas marocain », Techniques \& Culture [En ligne], 38 | 2002, mis en ligne le 11 juillet 2006, consulté le 29 septembre 2022. URL : http://journals.openedition.org/tc/230 ; DOI : https://doi.org/10.4000/tc.230

Ce document a été généré automatiquement le 29 septembre 2022.

Tous droits réservés 


\title{
Gérer les aléas. Les sociétés du Haut Atlas marocain ${ }^{1}$
}

\author{
Martine Garrigues-Cresswell et Béatrice Lecestre-Rollier
}

Dans les sociétés de montagne, au Maroc, l'aléa est une donnée permanente, toujours présente. Et ce, qu'il s'agisse d'aléas d'ordre climatique (le gel qui brûle les jeunes pousses, l'enneigement qui ne permet plus aux troupeaux d'accéder aux parcours pour se nourrir, les pluies diluviennes qui font déborder les torrents et dévastent les champs ou encore la sécheresse estivale qui limite l'irrigation des cultures), ou bien d'aléas d'ordre socio-politique (la rupture de relations d'al-liance ou de coopération entre groupes ou encore les fluctuations démogra-phiques). Il ne s'agit pas d'accidents, ou de catastrophes imprévisibles, mais bel et bien de contretemps, redoutés certes, mais néanmoins attendus dans la mesure où chacun sait qu'ils se produisent régulièrement. Nous ne sommes pas là dans le domaine de l'inconnu, de l'impensable, mais dans celui du connu. Ce qui est prévisible, c'est que cela va arriver même si on n'en mesure pas l'ampleur. Ce qui est imprévisible, c'est la date à laquelle cela arrivera: peut-être demain, dans quelques mois, l'année prochaine ou dans plusieurs années seulement; cela peut se produire plus ou moins régulièrement -parfois deux années consécutives -, ou bien ne pas se produire pendant plusieurs années tant sont importantes les variations d'une année sur l'autre, climatiques comme socio-démographiques. Le risque est quelque part tapi dans l'ombre, latent, et il faut être prêt à y faire face à n'importe quel moment.

2 La société tout entière s'organise, se structure même, au regard de cette donnée, c'està-dire moins par rapport aux périodes de plénitude qu'en fonction des risques de pénurie. Aussi les mécanismes sociaux ne se comprennent-ils que sur la longue durée; on ne saurait envisager de les décrire à partir de l'observation d'un seul cycle annuel. Ils impliquent des contraintes collectives fortes tant à l'échelle familiale que villageoise, ainsi que dans les relations inter-villageoises, voire intertribales du fait de la nécessaire complémentarité de milieux divers. Mais ils exigent dans le même temps une grande souplesse d'adaptation, qui permet de répondre rapidement aux aléas, prévisibles car probables, imprévisibles car imprécis et hypothétiques. Nous brosserons 
un panorama général -non exhaustif- des principales situations de risque telles que la société les appréhende et autour desquelles elle se construit. Nous évoquerons d'abord les aléas relatifs au milieu dit naturel, puis ceux qui relèvent du milieu humain, sachant toutefois qu'il s'agit là d'un découpage artificiel, les uns étant indissociablement liés aux autres. En effet, n'importe quel fait tient compte et engage l'ensemble des autres faits dans la façon dont les groupes sociaux assurent tout à la fois leur propre reproduction et celle de leur milieu. Nous nous fondons sur nos propres données de terrain, recueillies dans les vallées du Haut Atlas central et occidental au sein des sociétés d'agro-pasteurs berbérophones ayt Bou-Guemez, ayt Abbas, Rherhaya, Ourika, Mesfiwa, et sur la littérature anthropologique relative à cette aire culturelle²

La complémentarité verticale

3 Le Haut Atlas marocain présente les caractéristiques de la plupart des milieux montagnards : un relief à fortes pentes qui limitent considérablement l'extension des cultures -concentrées dans les fonds de vallées (entre 1500 et $2000 \mathrm{~m}$ d'altitude environ) où coule l'eau; une grande dispersion des ressources, fonction de l'exposition, de l'altitude, des sols, des possibilités d'irriguer, etc.; de fortes amplitudes thermiques dues à la haute altitude; enfin, des sols arables pauvres. Dans ce milieu fortement marqué par la précarité, le système productif intègre l'ensemble des étages écologiques et des ressources disponibles aux différents moments de l'année. Le système de production est caractérisé par une grande diversification des produits agricoles (orge, blé, mil, seigle et maïs ${ }^{3}$ traditionnellement, auxquels s'ajoutent, ou se substituent désormais, pommes de terre et luzerne ainsi que des cultures maraîchères et légumineuses: navets, courges, oignons, haricots, lentilles, pois...), des produits arboricoles (noyers, maintenant également pommiers, voire pruniers, cerisiers, pêchers...) et des activités pastorales (élevage des bovins, des caprins, des ovins, parfois même des équidés, basse-cour). Toutes ces activités sont extrêmement imbriquées. Il s'agit d'utiliser toutes les possibilités de diversification et de complémentarité des ressources qui s'étagent sur des gradients bioclimatiques divers, et de trouver les aménagements les plus efficaces, techniquement comme socialement, pour mettre en valeur l'ensemble des espaces. On est là dans une logique de la verticalité propre aux groupes montagnards, mise en évidence par les auteurs qui ont analysé les échanges et les relations entre plaine et montagne, hautes et basses terres, et ce dans des sociétés aussi différentes que celles des Alpes ou des Andes, de l'Himalaya ou du Haut Atlas 4 .

Dans le Haut Atlas, les populations se sont toujours efforcées de constituer leurs finages perpendiculairement aux axes principaux des montagnes, depuis les pâturages de haute altitude jusqu'aux vallées encaissées et si possible, au-delà, jusqu'aux terres de culture et aux steppes pastorales des piémonts, voire de la plaine. On a montré qu'il existe «toute une série de formes mixtes de verticalité » (Garrigues-Cresswell 1987). Une même population peut occuper plusieurs étages écologiques, soit d'un seul tenant, soit de façon discontinue avec un territoire en montagne et un territoire séparé en plaine ou dans la zone de piémont. À travers la transhumance agropastorale, le groupe exploite ainsi directement plusieurs micro-niches à différents niveaux d'altitude.

Dans le même but, une population, confinée dans une seule zone, peut se spécialiser dans certaines activités agricoles et/ou pastorales, développant des relations commerciales, voire des alliances, avec des partenaires installés sur un territoire susceptible de fournir les ressources manquantes, en premier lieu des parcours d'hiver pour les troupeaux. Cette instauration de liens privilégiés est un phénomène ancien, et 
nombreux sont les montagnards qui ont hérité de leur père leur correspondant en plaine. De nouvelles relations s'établissent encore à présent, car les échanges économiques qui en découlent sont nombreux : échanges de produits complémentaires (noix, maïs, animaux, aujourd'hui également pommes de terre et fruits, contre huile d'olive et céréales de la plaine), cadeaux réciproques sous forme de don et de contredon, octroi de droits de parcours en contrepartie d'une main-d'œuvre disponible, etc.

Une autre issue pour les montagnards est d'acheter ou de prendre en métayage des terres de culture en plaine. À certaines époques de l'année, les membres d'un groupe domestique se trouvent alors répartis sur plusieurs étages écologiques: l'habitation permanente du village ou les bergeries de haute montagne sur les parcours estivaux, enfin les parcelles en plaine ou sur le piémont.

7 L'idéal de verticalité peut même, en certaines circonstances, céder la place à un idéal d'autosubsistance qui permet aux populations de faire face aux aléas politiques. Car si le milieu physique est difficile, le milieu humain ne l'est pas moins. Si les groupes peuvent se répandre en plaine ou sur les piémonts, et donc compléter leurs ressources locales par celles de ces territoires différents, ils peuvent aussi se réfugier en altitude pour échapper à la menace du pouvoir central ou fuir la puissance d'autres groupes. Leurs stratégies spatiales et sociales se complètent et s'adaptent en permanence aux aléas climatiques comme aux vicissitudes politiques.

8 À ces contraintes habituelles des milieux montagnards, auxquelles plusieurs formes de verticalité apportent autant de réponses, s'ajoute ici celle d'un milieu semi-aride caractérisé par une très grande et très brusque variabilité -tant annuelle qu'interannuelle- des conditions climatiques. Si ces dernières autorisent le développement des activités agricoles et pastorales, la productivité est limitée par le froid hivernal, par la sécheresse estivale ainsi que par la nécessité d'une constante et rapide adaptation aux aléas : irrégularité des pluies, de la neige, du gel. Ces altérations brutales des conditions de la production ne sont pas toujours de l'ordre du « coup dur » dont on se remet plus ou moins facilement. Elles peuvent aller jusqu'à compromettre la reproduction de l'assise matérielle des groupes.

Pratiques et stratégies pastorales

9 Les pratiques intègrent l'ensemble des aléas susceptibles de survenir à un moment ou un autre, et, pour les comprendre, il faut considérer une dimension temporelle pluriannuelle, on l'a dit. Par exemple, l'élevage des petits ruminants - ovins et caprins- est strictement dépendant de la productivité des parcours pastoraux. Le petit bétail ne bénéficie que de façon ponctuelle des ressources issues de l'exploitation agricole variées mais peu abondantes-, qui sont prioritairement affectées aux bovins et aux équidés. Aux périodes charnières du cycle climatique annuel (jours de neige, soudure à la fin de l'hiver) ou du cycle physiologique des animaux (fin de gestation, lactation, jeunes non sevrés qui ne suivent pas encore leur mère au parcours), les animaux reçoivent alors une alimentation complémentaire : orge en grains, luzerne, foin... Les jeunes et leurs mères pâturent les pousses de céréales semées à la fin de l'été. Mais jamais les éleveurs ne sont capables de subvenir eux-mêmes bien longtemps aux besoins du cheptel ovin et caprin. Or, la productivité des parcours pastoraux est soumise aux conditions climatiques. S'il a plu abondamment, s'il a neigé sur les hauteurs, la flore sera riche et les parcours pourront engraisser de nombreuses bêtes pendant la saison estivale; si tel n'est pas le cas, la flore sera pauvre et ne suffira pas aux besoins d'un cheptel important. Et si le froid et la neige s'abattent durablement sur 
la vallée, peu de bêtes résisteront aux rigueurs de l'hiver. La vulnérabilité du sys-tème alimentaire est donc très grande. Elle rend compte des fluctuations importantes de la productivité du cheptel dont les effectifs peuvent passer du simple au double d'une année sur l'autre.

La totalité des pratiques et des stratégies pastorales répondent à cette variabilité des conditions de production. Les éleveurs n'y font face que grâce à une grande souplesse d'adaptation: vente précoce des animaux qui n'ont que peu de chance de survivre à l'hiver, puis rachat de femelles jusqu'à épuisement de la trésorerie disponible dès que la conjoncture s'annonce favorable, achat d'aliments complémentaires dans la limite de leurs disponibilités financières, transhumance hivernale vers la plaine ou les piémonts, accords de gardiennage, associations sur le bétail et accords pastoraux qui permettent de multiplier l'accès à des parcours ainsi que d'ajuster en permanence et de façon optimale la taille du cheptel à la productivité des pâturages. Des accords temporaires de gardiennage, par exemple, résolvent les problèmes posés par l'ajustement de la maind'œuvre familiale aux contraintes agropastorales. La main-d'œuvre est précieuse l'été dans la vallée, à l'époque des gros travaux agricoles. Nombre de petits troupeaux sont alors confiés, le temps des moissons ou pour la durée de l'estivage, à des éleveurs importants qui emmènent les bêtes avec leur propre troupeau sur les alpages, à charge pour eux de négocier, si nécessaire, avec l'ensemble des ayants-droit, l'autorisation de pâture pour des bêtes étrangères ${ }^{5}$. En contrepartie sont dues des journées de travail ou bien un coup de main au plus fort des travaux agricoles, pour le battage, par exemple. Quelquefois, un jeune garçon de la famille accompagne les bêtes confiées et seconde utilement les bergers qui ont en charge l'ensemble des animaux. Le bétail est ainsi l'enjeu d'une multitude de relations, tant économiques que sociales et politiques. Différentes formes de contrat font intervenir le gardiennage, le partage des bergeries, les échanges de services, les associations sur le bétail avec répartition des produits entre propriétaires et gardiens, les accords pastoraux qui prévoient l'ouverture ou au contraire la fermeture des parcours aux troupeaux étrangers en fonction de la qualité de la flore. Il s'agit là de pratiques courantes qui participent de tout un réseau très dense de relations sociales. Car si l'intérêt économique de ces relations est indéniable, leur importance sociale ne l'est pas moins. On entrevoit le passage de rapports de solidarité économique à des rapports de clientèle générateurs d'inégalités politiques.

11 Plus on entre dans les détails, plus se révèle la complexité de pratiques pastorales multiples, dont la logique est la recherche permanente et toujours renouvelée de la meilleure adaptation possible non seulement aux conditions climatiques, à la qualité des parcours, mais en outre à la productivité de l'agriculture -si les récoltes sont bonnes, une part plus importante peut être affectée aux besoins alimentaires du cheptel-, à la main-d'œuvre familiale disponible, aux relations des groupes à l'espace pastoral, etc. Et plus on multiplie les paramètres, plus on perçoit l'adéquation optimale réalisée entre ces différents facteurs. En hiver, un des risques majeurs pour le troupeau est un fort enneigement qui empê-cherait les animaux d'accéder aux parcours; la seule solution consiste alors à couper en forêt du fourrage foliaire (feuillage du chêne vert et du genévrier, essentiellement), afin de le rapporter au troupeau confiné à l'étable. Les bergers et les hommes disponibles se chargent de ce travail difficile, entravé par le froid et la neige. C'est pourquoi les versants forestiers les plus bas, les plus proches des villages, sont en général réservés à cette fin. Ils sont ordinairement mis en défens, c'està-dire soustraits à toute coupe de bois. La mise en défens ne peut être levée -pour quelques jours seulement- que pour les coupes de fourrage foliaire et quand 
l'épaisseur de la couverture nivale est telle que ni les hommes ni les bêtes ne peuvent se déplacer sur les hauteurs. La gestion des massifs forestiers est fonction de cet aléa. Bien d'autres mises en défens existent quant aux coupes de bois d'œuvre, de bois pour le feu, etc., variables selon les groupes, selon l'importance de leurs espaces boisés, selon l'emprise des servitudes communautaires; mais tous les groupes qui gèrent encore collectivement la forêt tiennent compte de cette contingence-là ${ }^{6}$. La coupe de feuillages reste le dernier recours pour pallier les rigueurs brutales de l'hiver, à condition toutefois que les massifs forestiers proches soient assez fournis pour supporter quelques jours de coupe. Une telle contrainte pèse donc particulièrement: elle oblige les gens à de longs et pénibles déplacements en haute montagne, vers les massifs boisés lointains, pour y effectuer habituellement leurs coupes de bois, réservant les massifs les plus proches à un usage tout à fait occasionnel, de courte durée, et qui peut fort bien ne pas advenir pendant plusieurs années consécutives. La ponction des hommes sur la forêt est alors très importante -pour un troupeau moyen de 150 à 200 têtes, ce sont deux ou trois chargements de mule, soit six ou huit charges d'homme, qu'il importe de prélever quotidiennement sur la forêt-, et cet apport ne peut être que temporaire.

Si la neige persiste, si le froid s'installe durablement, les éleveurs doivent entreprendre la transhumance hivernale en plaine ou sur les piémonts, afin que les troupeaux -au moins les animaux les plus fragiles- échappent aux rigueurs de l'hiver. Selon les groupes, la topographie ou l'histoire des relations intertribales, les voies de la transhumance mènent au nord ou au sud de la grande chaîne de l'Atlas. Ainsi, les ayt Bou-Guemez et les ayt Abbas se dirigent majoritairement vers les piémonts sud, vers le jbel Sarhro qui borde le Sahara, sur les terres de parcours des Imeghran, des Imgoun, des ayt Atta, des groupes qui se situent sur le versant sud de la chaîne atlassique. Seuls quelques groupes parmi eux avaient traditionnellement établi aussi des voies vers le nord jusque sur le Dir. Les Rherhaya, au contraire, s'orientent vers les régions nord d'Asni, Tahanaout, Amizmiz, Oumnast, Tameslouht et, au-delà, en direction des plaines de Chemaïa, de Chichaoua ou de Benguérir.

L'accès à des terres de parcours lointaines repose sur des réseaux d'échange avec les groupes installés en plaine et sur les piémonts. Il peut s'agir d'accords pastoraux passés dans le cadre d'une réciprocité et d'une complémentarité des échanges. Ou bien d'alliances matrimoniales - la carte des mariages exogames lointains recoupe celle des voies de la transhumance- qui sous-tendent des échanges de service et des dons mutuels variés. Traditionnellement, dans toute la partie centrale du Haut Atlas, la vie pastorale se fonde sur un double mouvement des troupeaux : une circulation estivale vers les hauts pâturages des pentes nord de la chaîne, qui concerne aussi bien les éleveurs établis dans les vallées du versant nord que ceux installés au sud, et un déplacement hivernal vers les piémonts sud de la montagne. La transhumance estivale concerne plusieurs centaines de milliers de bêtes : toutes celles qui fuient la sécheresse au sud et qui voisinent avec celles des lieux, encore plus nombreuses; elles s'accumulent sur les parcours les plus riches. Le mouvement hivernal vers le sud est moins important. Outre les troupeaux du sud qui regagnent leur base de départ, il entraîne une partie seulement des animaux du nord qui délaissent le froid et la neige pour des cieux plus cléments, jusque sur les parcours d'armoise du Sarhro. Une complémentarité s'est donc instaurée entre les éleveurs du Sud et ceux du Nord. Si les parcours les plus prisés, les beaux herbages situés dans les dépressions humides, les hautes combes, font l'objet de conventions pastorales précises, dont les plus 
importantes don-nèrent lieu à l'élaboration d'une charte de la transhumance initiée par les autorités coloniales, la plupart des échanges se font dans le cadre d'une réciprocité des mouvements. Les groupes ou les familles qui perpétuent aujourd'hui la tradition de la transhumance ${ }^{7}$ se déplacent, l'hiver, au sud, sur les terres de parcours des groupes qui profitent, l'été, de l'accès à leurs propres pâturages au nord. Des alliances politiques, qui se poursuivent désormais sous des formes nouvelles, renforçaient jadis ces réseaux d'échange historiquement constitués (cf. infra). Dans la partie occidentale de la chaîne atlassique, chez les Rherhaya en particulier, les réseaux d'échange s'organisent autour de liens privilégiés tissés de personne à personne, entre partenaires de montagne et de plaine. Lorsque ces liens sont consolidés par des mariages, alors les échanges prennent place au sein d'un vaste système de rapports réciproques, beaucoup plus flexible entre parents qu'entre simples partenaires économiques. Ils tendent notamment à s'effectuer sous forme de dons et contre-dons, et à être ajustés sur le long terme et non dans l'immédiat. Les alliances se font toujours dans le même sens : une jeune fille d'un groupe familial établi en montagne est donnée en mariage à un homme installé en plaine. Les animaux sont ainsi descendus pendant l'hiver chez une sœur ou chez les enfants d'une sœur.

14 N'importe quelle pratique pastorale, aussi anodine qu'elle paraisse, a des répercussions sur l'ensemble de l'organisation sociale. L'enchevêtrement des facteurs est extrême : toute décision intègre un grand nombre de paramètres. Ce n'est qu'au prix d'une très grande souplesse d'adaptation que les éleveurs, qui sont aussi agriculteurs, réussissent à mettre en valeur un milieu difficile, où les aléas climatiques sont une donnée fondamentale de leurs choix techniques, économiques et sociaux. Les déplacements des animaux, s'ils obéissent à un rythme saisonnier et suivent des itinéraires connus parcours journaliers sur les pentes forestières proches des villages d'octobre à mai-juin, montée et séjour sur les pâturages de haute altitude de mai-juin à octobre, avec souvent des possibilités de séjour dans des bergeries intermédiaires entre le village et les alpages, éventuellement transhumance hivernale en plaine ou sur les piémonts-, n'en sont pas moins totalement dépendants de la pluviométrie qui fait la richesse des parcours, du froid et de la neige qui entravent les mouvements, ainsi que des variations qui affectent l'exploitation agricole et le groupe domestique. La complémentarité des espaces n'est pas un vain mot. Les parcours font partie intégrante d'un vaste ensemble qui s'étend sur des étages différents et complémentaires allant des fonds de vallée irrigués aux espaces asylvatiques des hauts plateaux et des crêtes en passant par les versants forestiers et, au-delà, aux steppes pastorales des piémonts, voire aux parcours sur chaume de la plaine. Le pasteur, à la recherche permanente du meilleur équilibre, forcément précaire, entre les ressources pastorales et les moyens dont il dispose, doit être prêt à modifier à tout instant sa stratégie. Ne dit-on pas que le grand pasteur (aksab, pl. iksabn) est partout à la fois : il veille, dès qu'il en a la possibilité, à séparer le troupeau afin de respecter les exigences propres à chaque espèce ${ }^{8}$ et de limiter les risques d'épizootie ou de mauvais pâturages, à constituer temporairement de petits troupeaux (jeunes, femelles gestantes ou ayant mis bas, bêtes fatiguées, blessées) qui se déplacent sous la garde des jeunes enfants de la famille en fonction des ressources du terroir irrigué (céréales en herbe, prairie, chaumes après les moissons, fanes des pommes de terre, etc.), à négocier des accords de gardiennage ou contracter des associations sur le bétail pour être en mesure, le moment venu, de répondre de façon optimale aux contraintes occasionnelles; il fait régulièrement la tournée de ses animaux répartis ainsi en différents lieux, ravitaille ses bergers, les conseille sur les 
itinéraires à suivre, ramène éventuellement les bêtes qui seront vendues sur le souk; ou encore, il s'enquiert sur les marchés ou auprès des autres éleveurs de la qualité des parcours afin d'être le premier à pousser ses bêtes sur les meilleurs herbages, etc.

Un équilibre instable

L'équilibre, instable, provisoire, ne s'établit qu'au prix d'une importante flexibilité. Des dysfonctionnements -mais peut-on encore les qualifier ainsi- surgissent régulièrement, telle l'obligation de récolter avant maturité pour que les animaux qui ne sont pas au parcours puissent pâturer sur les chaumes ou bien parce que les premiers froids de l'automne compromettent la deuxième récolte. Le choix entre diverses options est préservé jusqu'au dernier moment. La destination finale de certaines cultures - pour l'alimentation humaine ou animale et, dans ce dernier cas, pâturée sur pied ou engrangée pour l'hiver- n'est décidée qu'en tout dernier lieu et en tenant compte de l'ensemble des aléas : conditions climatiques, besoins du cheptel, besoins humains, disponibilité en main-d'œuvre, disponibilités financières, prévisions à court et à long terme, etc. L'orge est l'exemple même d'une culture polyfonctionnelle qui produit aussi bien du grain pour la consommation humaine et animale que du fourrage vert, de la paille et jusqu'à de mauvaises herbes soigneusement récupérées. C'est pourquoi elle tient toujours une place importante dans les assolements, malgré certaines évolutions actuelles en faveur d'autres cultures. Les semences de l'orge (tim $\Delta i n$, tum $\Delta i n$ ) et de l'orge fourragère (agulas, ag $\left.{ }^{w} l a s\right)$ sont identiques. La distinction terminologique recouvre simplement une différence quant à la destination du produit : grain ou fourrage. L'orge semée afin de récolter du fourrage fait l'objet de semis plus denses, d'irrigations plus fréquentes, sans aucun désherbage. Cependant, le choix de sa destination finale reste ouvert le plus tard possible : semée en vue de récolter du grain, elle peut être préalablement pâturée ou fauchée pour du fourrage, ou bien l'inverse. La date du semis varie d'octobre, si la pluviométrie est convenable, à janvier, si l'hiver est froid et sec. Semée vers la mi-août et fauchée en novembre, elle constitue une réserve de fourrage pour le bétail en hiver. Mais elle peut aussi être pâturée sur pied de l'automne au printemps. C'est donc une culture d'utilisation très souple, qui peut être cultivée en cycle long ( $a m n z u)$ ou court $(a m a \Delta u \Delta)$. Le maïs, bien que dans une moindre mesure, est également une culture qui peut produire à la fois du grain pour la consommation humaine et animale, du fourrage ainsi que des tiges et des feuilles pour le bétail.

Les arrangements culturaux sont donc toujours orientés vers la recherche de l'adaptabilité optimale à la conjoncture du moment. C'est quand il n'est plus possible de reculer davantage le choix qu'est prise la décision d'ensemencer les terres bur ${ }^{9}$ ou de faire deux récoltes successives sur une même parcelle, et ce, compte tenu de la pluviométrie, des ressources en eau pour l'irrigation, des besoins du cheptel et du groupe domestique. Tout se tient, toute option se répercute sur l'ensemble du système productif et engage la totalité de l'organisation sociale; au niveau de l'espace privé comme de l'espace public, de la plus petite parcelle cultivée au fond de la vallée jusqu'aux vastes parcours intertribaux de haute altitude et, au-delà, aux routes lointaines de la transhumance ainsi qu'à l'échelle du temps -saisonnière, annuelle, pluri-annuelle, intégrant les bonnes comme les mauvaises années-, toute décision se présente sous la forme d'une alternative relative aux aléas du milieu, physique comme humain. Comme le souligne Alain Bourbouze (1982), les systèmes de production du 
Haut Atlas ne réalisent-ils pas un équilibre optimal sous l'effet des multiples contraintes qui leur sont appliquées?

Le système des leff-s dans le Haut Atlas occidental

Il y a aussi d'autres aléas : les "risques humains ", d'ordre politique et démographique. Sans entrer dans trop de données historiques, rappelons que le Haut Atlas marocain, avant l'instauration du protectorat français en 1912 et dans les premières décennies qui ont suivi, était le lieu d'une grande instabilité politique. Les conflits y étaient nombreux, pour des motifs tant internes qu'externes, en liaison avec le Makhzen, le pouvoir central. Différentes institutions permettaient aux populations de survivre pendant les périodes de conflit. Retenons notamment les greniers-citadelles ou greniers collectifs fortifiés, les trêves instaurées pour la tenue des marchés, les lieux sacrés -et donc protégés- comme les sanctuaires, enfin les pactes d'alliance et de protection sur lesquels nous centrerons ici notre attention.

Depuis les travaux de Robert Montagne (1930), le système des leff-s au Maroc est bien connu. Le terme arabe de leff renvoie au terme berbère de amqqun, tandis qu'est usité en Algérie le terme de soff. Soulignons aussi que dans la langue actuelle, les habitants $\mathrm{du}$ Haut Atlas n'emploient généralement aucune de ces dénominations, alors qu'y demeure bien présente la distinction entre groupes appartenant à des ligues différentes. Chez les Rherhaya, par exemple, les ayt Fademt et les ayt Tzgout sont désignés respectivement par leur nom propre. Ainsi, ces groupes montagnards, comme de nombreuses sociétés du monde arabo-berbère, se partagent en deux grandes alliances ou ligues politiques. Dans cette région, ce n'est pas la tribu entière mais chacune de ses fractions qui se rattache à l'un des deux leff-s. Chacune entre isolément dans l'un ou l'autre parti et les leff-s se distribuent en un immense échiquier à deux couleurs. Traversant la division en tribus, l'institution des leff-s permet, en temps de guerre, d'équilibrer les forces en présence et donc de limiter la portée des conflits. Si l'accent a été mis sur le rôle politique de cette institution, en revanche on n'a pas, ou pas assez, réfléchi à ses fonctions économiques et d'adaptation écologique. Pourtant, Robert Montagne lui-même, alors que sa réflexion portait essentiellement sur le domaine politique, suggérait déjà cette idée :

«Entre les cantons du même leff - ceux de la haute montagne et ceux du Dir-, s'établissent aussi des relations économiques d'une grande importance. Dans les premiers se trouvent de riches pâturages d'été, des récoltes de noix, de la laine en abondance, mais on y manque d'orge et de maïs, car la terre est rare dans les vallées encaissées. Au contraire, les cantons du Dir ont à leurs pieds d'immenses espaces qui peuvent se cultiver en bour ainsi que des champs irrigués, mais les troupeaux ne peuvent subsister en plaine au cœur de l'été. Ces raisons suffisent à créer des échanges importants : les troupeaux des Seksawa descendent en plaine à l'automne, ceux des Mzouda, des Dwiran et des Gedmiwa montent vers les sommets à la fin du printemps. Mais chacun ne se confie qu'à ses frères de leff afin d'obtenir protection pour ses biens si la guerre venait à éclater. » (Montagne 1930 : 191-192) ${ }^{10}$.

Jean Dresch, qui parcourut le Haut Atlas, a établi des cartes et apporte dans son Commentaire quelques nuances à la théorie de Montagne :

«Malgré leur stabilité, les leffs ne sont pourtant pas immuables [...]. Il est non moins difficile d'établir la correspondance entre des leffs de nom différent [...]. Dans d'autres régions, enfin, il est impossible de trouver trace de leffs. » (Dresch 1941 : 9-10).

20 Mais il ajoute quelques lignes plus loin : 


\begin{abstract}
«Malgré une confusion apparente, qui résulte souvent de troubles, plus ou moins récents, il n'est pas rare de constater une coïncidence entre ces alliances et des intérêts économiques communs : des vallées entières et leurs débouchés sont tenus par le même leff (ex. : Aït Bkhar et Taskemt, Aït Chaib et Aït Messaoud, les Seksaoua); ou bien des groupements du même leff tiennent toute la zone de bordure, les sources des vallées, les terrains bour et les pâturages des plateaux (ex. : fractions bordières des Irguiten et des Mentaga); d'autres tiennent au contraire les hauts massifs, comme les Aït Iberdaten, Aït Ouardouz, les hauts villages de l'Anougal sur le versant Nord du massif Erdouz-Gourza, les villages des Aït Tidili situés au pied des hauts pâturages des montagnes du Zat, à moins qu'au contraire on ne retrouve, sur chaque versant, des alliés qui gardent les passages; d'autres alliance paraissent s'expliquer par le partage de l'eau ou de terrains. » (Dresch 1941 :10).
\end{abstract}

21 Nos propres observations dans le Haut Atlas occidental, que ce soit chez les Rherhaya, les Ourika ou les Mesfiwa, ont montré que la répartition entre les deux leff-s intervenait dans la gestion de l'irrigation, de l'accès aux prairies d'altitude ou de la transhumance en plaine (Garrigues-Cresswell 1985, 1987).

22 Ainsi, le relevé du système d'irrigation du Haut Rherhaya fait apparaître que la prise d'eau sur le torrent d'où dérive un canal destiné à irriguer les terres d'un village se situe fréquemment non pas sur le finage du village concerné mais sur le territoire du village situé en amont, lequel appartient parfois à une autre fraction, rattachée à l'autre leff. Les villageois sont parfaitement conscients de ce fait «stratégique » et de ses enjeux. Ils indiquent spontanément qu'en cas de guerre, couper l'eau du canal du village d'Ayt Souka (leff des ayt Fademt), qui prend son embranchement dans le territoire d'Imlil (leff des ayt Tzgout) sur le Haut Rherhaya, risquerait d'entraîner la riposte immédiate des habitants de Wanskra (ayt Fademt), lesquels couperaient alors l'eau que le village de Tamguist (ayt Tzgout) fait dériver sur leur propre territoire dans la vallée de l'Imenan.

De manière similaire, les alliances de leff-s et leur enchevêtrement ont pu contribuer à limiter la portée des incidents et à éviter l'extension des conflits survenant sur les grands pâturages collectifs d'altitude pendant l'estivage, comme l'Oukaimeden et le Yagour, où séjournent pendant plusieurs semaines des centaines de bergers relevant de fractions et même de tribus différentes. Ils viennent en cela conforter le rôle pacificateur des descendants des saints, Sidi Fars pour l'Oukaimeden et Sidi Bou Jemaa pour le Yagour, qui, par leur caractère sacré, assurent la protection des pâturages (Gellner 1969).

24 Enfin, rappelons que les ressources des hautes vallées ne permettent pas d'assurer l'alimentation des ovins pendant la période d'enneigement. Il est donc nécessaire de trouver des parcours chez des "partenaires " en plaine ou sur le piémont, dans des lieux situés à plusieurs dizaines de kilomètres. L'appartenance au même leff facilite alors l'établissement de ces rapports privilégiés entre montagnards et villageois de la plaine. Et la répartition des villages traversés lors de la conduite du troupeau entre les deux leff-s opposés octroie également une certaine sécurité, comme on l'a vu à propos des droits sur l'eau. Ajoutons enfin que si la plupart des mariages ont lieu au sein des fractions, les mariages lointains s'effectuent, eux, de préférence entre membres du même leff.

25 Ainsi, le système des leff-s, outre ses fonctions politiques - et nous entendons par là aussi bien les alliances guerrières que la sécurité des sources, des biens et des troupeaux en temps de paix-, jouait un rôle important dans la vie écono-mique en 
venant conforter les accords concernant la répartition de l'eau ou des parcours de transhumance. Il procurait également des partenaires commerciaux, parallèlement aux échanges s'effectuant sur les marchés.

Les pactes de colactation dans le Haut Atlas central

Dans le Haut Atlas central, si les alliances de leff-s sont connues, elles ne présentent pas du tout la même permanence; plus fluctuantes, elles se modifient selon les situations conjoncturelles. Elles ne peuvent donc pas remplir les mêmes fonctions que dans le Haut Atlas occidental. En revanche, il existe une autre institution, inconnue, semble-til, dans le Haut Atlas occidental -nous n'en avons pas trouvé de trace chez les Rherhaya et Berque ne la signale pas non plus chez les Seksawa. Il s'agit des ayt †адa qui sont unis par un pacte d'assistance mutuelle. La forme la plus remarquable consiste en un échange de lait. L'une des premières mentions en est faite par Edward Westermarck en 1914 quand il évoque les interdits de mariage :

«There are, moreover, prohibitions of intermarriage referring to some special tribes, subdivisions of tribes, or villages, in connection with other peculiarities in their social relations. Thus there is so-called tada, or brotherhood, between the Infduak (Ftuâka) and Igliwa in the Great Atlas, which implies that no marriage is allowed between them, and also that if a member of one of this tribes takes refuge with the other tribe, he cannot be touched. (Westermarck 1972 : 57).

Outre les tribus déjà citées, Westermarck a rencontré cette même institution chez les ayt Nder, les ayt Yusi et les ayt Sadden, avec des obligations encore plus nombreuses: interdit matrimonial, inviolabilité des refuges, obligation d'hospi-talité, exigence de respect se traduisant par l'interdiction de prononcer de mau-vaises paroles ou de mentir, possibilité d'arbitrer des querelles, etc. Une seule res-triction: cela n'impliquait, semble-t-il, aucune obligation d'assistance en cas de conflit armé.

28 À peu près à la même époque, l'institution est observée chez les Zemmour par le capitaine Coursimault qui écrit une brève note sur la ttatta. Il la définit comme « une sorte d'alliance qui revêt un caractère religieux et sacré » (1917: 261). Elle était autrefois pratiquée entre deux individus mais à l'époque de ses observations, elle se pratique entre tribus ou fractions de tribus. Cette alliance, nous dit-il, est « chose grave et régie par des préceptes rigoureux que l'on ne transgresse pas impunément, Dieu luimême se chargeant d'infliger la punition. [...] Ceux qui sont ainsi liés se doivent en toute circonstance un mutuel appui» (Coursimault $1917: 261$ ). L'auteur indique ensuite le rituel destiné à conclure le pacte: sacrifice d'un mouton suivi d'un repas communautaire, puis tirage au sort des sandales des participants, regroupées en deux tas -chaque participant ayant remis une sandale. L'homme le plus âgé tire simultanément une sandale de chaque tas et leurs propriétaires respectifs se trouvent alors personnellement unis par le pacte d'alliance.

Georges Marcy, dans l'article qu'il consacre à cette forme d'alliance chez les Berbères du Maroc central, apporte de nouveaux éléments. Tout d'abord, concernant l'étymologie du terme de tâd'a ou tât'a (selon les parlers berbères), qui paraît signifier "l'action de prendre le sein", "d'être allaité». Il s'agit d'« une sorte d'alliance intertribale, à caractère magico-religieux très prononcé, qui -semble-t-il- se concluait essentiellement, à l'origine, par le recours au procédé symbolique de la colactation » (Marcy 1936: 957-958). L'auteur y verra d'ailleurs, à juste titre, une marque de l'importance des liens de la parenté maternelle chez les Berbères. En revanche, nous n'irons pas jusqu'à le suivre dans les conséquences qu'il en tire lorsque, marqué par la pensée évolutionniste de son époque, il y voit un «vestige » de la parenté maternelle 
qui aurait selon lui précédé la patrilinéarité chez les Berbères. Mais ne nous attardons pas sur ce point qui nous mènerait à un tout autre débat. Georges Marcy $(1936$ : 967) décrit les détails précis du rite tel qu'il a été conservé chez les ayt Mt'ir :

«On choisit, parmi chacune des deux parties, sept femmes nourrissant un enfant, dont on prélève et mêle le lait dans un bol qui circule ensuite de main en main et où chaque individu présent trempe à tour de rôle ses lèvres. »

Il indique que l'institution de liens individuels contractés solennellement entre les membres de deux fractions se retrouve dans toutes les sociétés berbères du Haut Atlas central, jusque chez les ayt 'At't'a sous des formes légèrement dif-férentes et atténuées "par le moyen d'un tirage au sort effectué avec une pièce de vêtement, sandale ou turban, ou même de simples bûchettes de bois " (Marcy 1936:967), et avec un repas communiel. Le pacte d'alliance comporte l'obligation mutuelle d'assistance, de même qu'une interdiction formelle d'inter-mariage, un devoir d'hospitalité et de protection pour la personne de son $u$-tâd' $a$ et ses biens lorsqu'il est de passage sur le territoire de la tribu -notamment en temps de guerre-, et l'interdiction de tout profit injustifié lors des transactions commerciales entre $u$-tâd'a. Il ajoute qu'à la sanction du manquement individuel aux obligations du pacte par imposition d'une lourde amende s'adjoint la crainte d'une malédiction divine. Dans la synthèse récente qu'il propose de ces pactes de colactation, C. Agabi retient les points suivants :

«La cérémonie consacrant la tad'a se déroulait en trois temps. La première phase consistait en un rassemblement autour d'un kerkour ou d'un sanctuaire. Ce rassemblement pouvait conserver le souvenir d'affrontements ou de rivalités anciennes, reproduits sous une forme ritualisée [...] La seconde phase, qui est la tad'a proprement dite, est un repas consommé en commun par les deux groupes [...]. Le couscous du repas était arrosé du lait des femmes des deux groupes. Dans certaines tribus le lait était remplacé par le miel, mais il est sûr que, comme son nom l'indique, la tad'a, assimilée à la tétée du nourrisson, était à l'origine une vraie colactation afin que les membres des deux tribus ou fractions se sentent devenus frères. [...] la troisième phase, connue aussi bien chez les Zemmour que chez les Ayt Atta, est intimement liée au repas communiel et se déroule dans les mêmes lieux; c'est le tirage au sort des chaussures. Les sandales droites des participants forment deux tas cachés chacun par un burnous. Les notables tirent simultanément une sandale de chaque tas, leurs propriétaires se trouvent alors personnellement unis par le pacte de fraternité, quels que soient leurs niveaux sociaux. Désormais, pour le frère d'alliance, la personne de l'u-fâa'a est sacrée en tout lieu et quelles que soient les circonstances. Ainsi l'alliance collective déterminée entre les deux groupes dans la colactation communielle se trouve confortée par les multiples fraternités individuelles consacrées par le tirage au sort des sandales. » (Agabi 1994 : 2 047).

31 Les études sur les Ihansalen (Gellner 1969), sur les ayt Atta (Hart 1981), sur les ayt Abbas et les ayt Bou Guemez (Lecestre-Rollier 1992), sur les ayt Goudjama (Amahan 1994), rapportent toutes l'existence des ayt tâda ${ }^{11}$, de même que la prégnance à l'époque actuelle de ces liens anciennement établis. Un tel pacte imposait des obligations importantes et créait des solidarités étroites entre ayt tâda. Ceux-ci se devaient aide et assistance mutuelle en toute occasion et notamment lors de leurs déplacements pastoraux et commerciaux. Cette alliance débordait largement le cadre des nécessités de la protection. Les ayt tâda comptaient les uns sur les autres en toute circonstance et notamment pour tout ce qui concernait la transhumance, qu'il s'agisse d'intercéder auprès des assemblées communautaires pour obtenir un droit de pâture sur les parcours ou de se procurer une bergerie, du fourrage pour les animaux ou tout autre produit indispensable, voire du numéraire. L'organisation de la transhumance 
hivernale des troupeaux de Bou-Guemez reposait ainsi sur cette vaste chaîne de solidarité et d'entraide qui couvrait d'immenses étendues.

Aujourd'hui encore, on constate la prégnance des relations entre ayt tâda. Si de tels attachements n'exigent plus les précautions et les engagements naguère requis pour protéger des alliés qu'ils unissaient contre les violences, ils n'en demeurent pas moins vivaces et sous-tendent toujours bien des rapports entre familles jadis liées de la sorte. Les hommes du Sud qui arrivent le samedi à Bou-Guemez pour le souk du dimanche à Tabant sont reçus chez les fils des ayt tâda de leurs pères. Réciproquement, les ayt Bou-Guemez qui, à l'arrivée dans un douar étranger, se présentent comme les fils des ayt tâda sont certains d'être bien accueillis. Ils comptent sur ces familles autrefois alliées, aujourd'hui amies, pour se procurer des produits frais (légumes, œufs, menthe...), de la paille pour leurs bêtes ou entretenir certains réseaux anciens de transhumance. Certes, les liens ne se font plus de fraction à fraction, mais sont devenus strictement des liens de personne à personne, encore que dans ces sociétés où l'honneur familial est si important, ce soient aussi les frères et les fils qui doivent impérativement accueillir, héberger, aider celui que l'on appelle maintenant un ami :

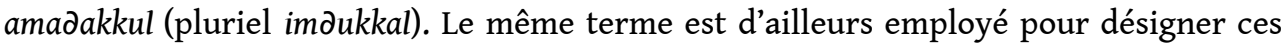
liens privilégiés dans le Haut Atlas occidental -domaine de prédilection des leff-s- et dans le Haut Atlas central -domaine de prédilection des ayt tâda. Il traduit cette relation particulière faite tout à la fois de camaraderie, d'amitié, de partenariat et qui implique surtout des obligations réciproques.

Dans le Haut Atlas occidental l'institution des leff-s, et dans le Haut Atlas central les pactes de fraternité, permettent donc l'accès à des parcours, ainsi que des échanges de biens, lesquels prennent souvent la forme de cadeaux réciproques. Au-delà de cette fonction commune, remarquons que ces liens privilégiés se créent, dans le premier cas en instaurant une relation d'alliance matrimoniale préférentielle -tendance à l'endogamie à l'intérieur d'un même leff-, et dans l'autre cas en créant une parenté élective -fraternité de lait et exogamie. On retrouve bien ici ce qui semble être une caractéristique des sociétés de l'aire d'influence arabo-musulmane, à savoir des relations complexes et subtiles entre consanguinité et affinité, dont le mariage dit "mariage arabe» constitue l'expression archétypale. Nous allons retrouver cette distinction "parenté versus alliance » au travers des réponses de ces sociétés aux aléas démographiques.

Le mariage uxorilocal et la captation de gendres

Dans ces sociétés à idéologie fortement patrilinéaire -même si elles présentent un cognatisme assez marqué, accordant une place importante à la parenté utérine-, il est essentiel d'assurer la perpétuation de son lignage. Or, dans les montagnes et à l'exception des lignages chorfa ou des lignages maraboutiques ${ }^{12}$, les lignages sont démographiquement peu importants, cela en raison de leur faible profondeur généalogique. Ne comprenant souvent que quelques dizaines d'individus, parfois moins, un lignage risque de s'éteindre en l'absence de descendants mâles. Par ailleurs, une unité domestique qui ne comporterait que des filles ne pourrait pas pratiquer l'ensemble des activités agropastorales qui exigent une main-d'œuvre abondante, ni jouer un rôle quelconque au sein des assemblées villageoises. Au sein de la famille étendue, les hommes du foyer se répartissent les tâches : l'un se consacre aux activités agricoles, un autre est berger, un troisième peut s'efforcer d'obtenir par des travaux artisanaux ou salariés le numéraire indispensable pour se procurer les biens non 
produits localement; le chef de famille, enfin, totalement ou partiellement libéré des activités productives, peut se consacrer à la vie publique, qu'il s'agisse de participer aux délibérations des assemblées villageoises, d'accueillir l'hôte de passage ou de se rendre aux différents souks hebdomadaires qui se tiennent dans le Dir. Car c'est là le lieu où s'échangent les biens mais aussi les nouvelles; là se nouent des liens tant avec les habitants des autres vallées qu'avec ceux de la plaine; là enfin, on rencontre les représentants du Makhzen, caïds et khalifes. On l'a compris, seule une famille étendue, riche en hommes, peut diversifier ses activités et espérer jouer un rôle politique. En revanche, une famille pauvre en individus de sexe masculin parvient difficilement à assurer sa subsistance; en outre, son chef de famille ne peut pas espérer tenir une place dans la vie politique locale et sa "maison» risque alors de disparaître, d'abord symboliquement, puis réellement -en l'absence de descendant mâle. Pour remédier à cette situation, on s'efforce de " capter un gendre » en lui donnant comme épouse une fille de la «maison » et en détournant la règle de patrilocalité. La résidence du couple sera donc uxorilocale et le gendre perpétuera le lignage et la «maison » de son beaupère. Il s'agit parfois d'un berger recruté enfant ou adolescent, issu d'une famille pauvre, qui est élevé au sein du foyer et aide le père dans toutes ses activités. Une fois adulte, ce «fils adoptif» se voit offrir en mariage une des filles de la famille. Certes, il lui est théoriquement possible de s'émanciper après son mariage, mais, très fréquemment, cette alliance hypergamique qui, notons-le, va à l'encontre de la règle qui préside aux mariages courants, fait qu'il acceptera de perpétuer ainsi la "maison » de sa femme, plus prestigieuse que la sienne.

Cette forme de mariage se rencontre dans tout le Haut Atlas ${ }^{13}$. L'analyse qu'en donne Georges Marcy $(1941: 198)$ sous le terme d'amazzal nous semble tout à fait pertinente :

"[il] équivaut en fait à une véritable adoption de mâle majeur, bénéficiant au clan de la femme, et conclue par l'intermédiaire de la fille d'un chef de tente -ainsi donnée par son père en mariage-, ou de la femme veuve restée chef de foyer et qui a choisi de se remarier dans ces conditions pour assurer une meilleure gestion de son patrimoine en le confiant aux mains robustes et actives de l'amazzal. »

Les enquêtes que nous avons menées attestent la fréquence de telles unions, bien qu'il soit très difficile de les comptabiliser avec précision lors du recueil de généalogies, car la «mémoire généalogique » s'efforce de vite "oublier » cet écart à la stricte "règle agnatique ", même si certains ne manquent pas de le rappeler en cas de différend.

La protection par « jet du car »

Il pouvait aussi se produire qu'un lignage puissant procure sa protection à un homme contraint à l'exil suite à un bannissement, pour échapper à une vengeance de sang ou encore en raison de sa pauvreté. Cet homme sollicitait alors, par un sacrifice animal fait au nom de Dieu, la permission de s'installer auprès de son protecteur, laquelle ne pouvait lui être refusée. Ce rite est connu sous le nom de ${ }^{c}$ ar, d'un terme arabe qui signifie le déshonneur, la honte; mais, ainsi que le souligne Edward Westermarck (1926: 548-549) :

«The term car, though an Arabic word, is not used in this sense by the oriental Arabs. The practices to which it is applied are supremely prevalent among Berber, as well as Arabic-speaking tribes in Africa, and present a multitude of forms to which I have found no parallels in the East. "

Parmi toutes les formes de « jet du ${ }^{c} a r$ », retenons les principales. La première, que nous venons d'évoquer, implique un sacrifice animal accompli devant la tente ou la maison de celui dont l'étranger implore la protection. Le chef de famille deviendra alors 
personnellement responsable de sa personne comme de ses biens, tant qu'il demeurera sur le territoire tribal. Mais le sacrifice peut aussi se faire devant la mosquée, et c'est alors la responsabilité du village entier qui est engagée. De la même manière, la protection d'un saint peut être sollicitée en employant une simple formule, accompagnée le plus souvent de la promesse d'un sacrifice. Westermarck (1972 : 339; 1926 : 522) relate également le fait qu'un fugitif peut aussi se placer sous le 'ar d'une femme en touchant son sein -il devient alors son fils- ou en pénétrant chez elle pour se saisir de son moulin à bras, ce qui le met automatiquement sous la protection de son mari et de sa famille, car "The Shloh say, Tamgart zund agurram, A woman is like a saint » (Westermarck 1972 : 339).

Le caractère commun à toutes ces expressions $\mathrm{du}^{c}{ }^{c}$ ar semble bien se trouver dans la malédiction attachée à son non-respect et, de manière intimement liée, au déshonneur qui s'ensuivrait. Comme l'ont bien montré Pierre Bourdieu (1972) ou Raymond Jamous (1981), c'est le respect du sacré qui fonde la conception de l'honneur. Il est interdit au plus fort de refuser son aide au plus faible, mais il revient à ce dernier de solliciter l'aide. Il devra pour exprimer sa demande faire preuve d'humilité; cependant, cette apparente soumission du demandeur masque une réelle position de force, car le demandeur contraint en fait la personne à qui il s'adresse. Celle-ci ne peut lui refuser sa protection, sous peine d'encourir l'opprobre et la honte. Et l'humiliation rejaillit sur celui qui manque à sa parole, qui ne respecte pas ses engagements. Il n'est plus digne de considération ni de respect; il n'est plus considéré comme un homme d'honneur car, s'il est vrai que haram (domaine du sacré et de l'interdit) ${ }^{14}$ et hachuma (le respect et la honte provoquée par un comportement déplacé ou par une conduite risquant de porter atteinte à l'honneur) relèvent de deux registres distincts, ils ne sont pas pour autant complètement étrangers l'un à l'autre. Le respect de soi est le garant de l'honneur qui s'exerce sur les domaines du sacré, et donc de l'interdit. On se rappellera à ce propos l'analyse approfondie effectuée par Pierre Bourdieu (1972) de l'honneur kabyle, et notamment le fait qu'il met en relation le nif, c'est-à-dire le point d'honneur, la respectabilité et la hurma -au sens de sacré-, qui à la fois s'opposent et sont inséparables. La vigilance concernant l'image de soi destinée aux autres permet de préserver le sacré dans sa double acception: le sacré droit -celui des agnats et des fusils-, et le sacré gauche identifié à l'univers féminin, à la maison, monde du secret par opposition au dehors ou monde ouvert de la place publique, réservé aux hommes. "C'est en effet le respect du sacré droit, du nom et du renom de la famille agnatique qui inspire la riposte à toute offense contre le sacré gauche » (Bourdieu 1972 : 35).

Et nous retrouvons bien là une dernière forme $d^{c}{ }^{c} a r$ qui, débarrassée de tout rituel sacrificiel et apparemment -mais apparemment seulement- détachée de toute connotation sacrée, oblige un individu à satisfaire la requête qui lui est adressée. Cet usage, qui consiste à solliciter l'intervention de personnes influentes par le moyen du ${ }^{c}$ ar ou plus simplement en leur faisant honte, est toujours observable dans le Maroc actuel, que ce soit en milieu rural ou en milieu urbain. On doit bien constater qu'il y a là un élément de permanence, quelque chose qui relève de la longue durée chère à Braudel, ou des structures profondes si l'on pense à la première définition qu'en donnait ce voyageur éclairé qu'était Edmond Doutté lors de son premier périple dans le Haut Atlas en $1901:$ : [...] l'car: ce mot s'entend dans l'arabe parlé actuel de tout rite ou de tout acte qui engage l'honneur de celui vis-à-vis duquel on le fait en présentant une demande » (Doutté 1914 : 253). 
41 Il relate à ce propos un incident survenu dans son voyage au temps du «vieux Makhzen ", lors de son départ d'Imi n Tânoût, après qu'il eut obtenu avec beaucoup de difficulté de louer quelques mulets supplémentaires, car il n'était pas, à cette époque, sans risque de faciliter le voyage d'un chrétien. Le loueur, notable personnage de l'endroit, dont le fils les accompagne, invoque leur protection :

«[...] il nous crie pendant que nous nous éloignons "Ala 'ârkoum 'Ala 'ârkoum"; mot à mot : "Sur votre honte, sur votre honte !" Cette honte, ce câr, c'est la mise en jeu de notre propre responsabilité, c'est l'obligation pour nous de répondre de la sécurité de son fils, sous peine, non seulement de la déconsidération, mais des malheurs qui pourraient nous être suscités par la divinité. » (Doutté 1914 : 252).

Ces différents procédés, parmi d'autres, témoignent de la plasticité dont font preuve ces sociétés, mais cette souplesse est sous-tendue par des règles collectives fortes. Il est bien clair que celui qui vient s'installer dans ces conditions devra ensuite respecter le « contrat social » et les règles rigides qui s'imposent à tous, afin que soient le plus vite possible « oubliées » ses origines.

La force des liens contractuels

Nous avons donc rencontré deux formes de pactes : des pactes d'alliance et des pactes de protection, selon la distinction de Henri Bruno et Georges-Henri Bousquet (1946). D'un côté se trouvent les leff-s et les ayt †âda et leur forme moderne, les liens de partenariat dénommés imaukkal, amis. Ces pactes d'alliance et d'assistance mutuelle expriment une relation réciproque et paritaire de type égalitaire. Les liens collectifs entraînent des liens privés, qu'ils se développent sur le modèle de l'alliance matrimoniale ou sur celui de la parenté de lait. De l'autre côté se trouvent le ${ }^{c} a r$ et le mariage uxorilocal avec captation du gendre, assimilé à un « fils adoptif ». Ces pactes de protection expriment, au départ, une relation asymétrique et inégalitaire, mais aussi une relation "obligée" pour celui qui est apparemment en position de force : contrainte liée à l'honneur et au risque de honte dans la première situation, contrainte liée à des nécessités économiques et au maintien d'une puissance politique qui implique d'avoir des descendants dans la seconde. En outre, et sans doute est-ce un corollaire de ce qui vient d'être dit, la relation, inégalitaire au départ, a pour vocation d'affirmer une égalité dans un futur plus ou moins lointain. La parenté élective ainsi créée est censée, non pas s'ajouter, mais se substituer au déficit de parenté qu'elle pallie (n'oublions pas que le droit musulman ne reconnaît pas l'adoption). Les liens privés vont donc déboucher sur une reconnaissance collective.

Par ces différents procédés, sont établis des liens contractuels. Ils peuvent en principe être datés, excepté les leff-s qui pourraient se rattacher à une structure plus permanente des sociétés méditerranéennes : alliances bipartites en Espagne (Jamard 1972) ou en Sicile (Patai 1962), soff en Algérie (Bourdieu 1972) ou en Tunisie (Bédoucha 1987), bipartition des villages libanais (Cresswell 1970). Ces pactes sont des phénomènes multidimensionnels qui ont, bien sûr, des implications politiques en cas de conflit ou lors des périodes d'instabilité, mais qui ont aussi une dimension économique. Les sociétés du Haut Atlas, répétons-le, mettent en valeur un milieu montagnard difficile, semi-aride et aléatoire. Elles ne peuvent pas vivre en autarcie : l'accès à des terres de parcours, à des produits vivriers (pour combler le déficit céréalier, se procurer de l'huile, du sel, etc.), à des travaux salariés en plaine ainsi que la possibilité d'écouler des productions de la montagne (animaux, noix, charbon de bois) leur sont indispensables pour assurer leur subsistance et leur reproduction.

De l'actualité de ces pratiques 

d'abord assumé des fonctions politiques. Ils sont en accord avec certaines valeurs fondamentales des sociétés arabo-berbères, telles que haram et hachuma. Le sacré est attaché aux liens de parenté : parenté utérine tout autant que parenté agnatique -et nous retrouvons ici le fait que les sociétés berbères ont des pratiques cognatiques sous une idéologie agnatique-, parenté par le sang tout autant que liens d'affinité -et nous retrouvons là une des caractéristiques de ces sociétés, pour lesquelles filiation et alliance présentent un certain degré de confusion. Mais ce que nous avons voulu souligner, c'est la fonction économique de ces institutions, que ce soit pour la reproduction des groupes domestiques ou pour la reproduction de la société dans son ensemble, à l'échelle familiale comme à l'échelle collective. Car c'est, nous semble-t-il, ce qui fait la permanence de ces institutions dans le Maroc contemporain, bien longtemps après la pacification des régions montagneuses. L'esprit de celles-ci, loin d'avoir complètement disparu, se maintient. Ainsi en va-t-il des liens d'amitié ou de camaraderie, sans cesse réactivés, avec des partenaires lointains, et qui permettent tout à la fois d'accéder à des parcours pastoraux, d'acheter ou de louer des terres cultivables en plaine, d'obtenir des produits sans passer par le souk, toutes formes «traditionnelles » d'échange qui se maintiennent $-\mathrm{y}$ compris sous des apparences nouvelles. Ainsi également du développement de formes plus « modernes " d'échange, comme l'accueil des enfants de la montagne chez les partenaires de plaine afin qu'ils puissent accéder à des établissements scolaires, comme l'hébergement par les montagnards des urbains qui viennent l'été prendre quelques vacances ou des pèlerins qui se rendent aux lieux sacrés, comme l'aide à la recherche d'emploi, etc. La facilité des déplacements multiplie aujourd'hui les occasions de contact et donc le besoin de disposer de réseaux d'interconnaissance et d'entraide, ne serait-ce d'ailleurs que pour les nécessités de l'hébergement. Or, dans les formes anciennes comme dans les formes actuelles, c'est bien sur ces deux valeurs essentielles que l'on s'appuie pour parvenir à ses fins : le sacré et l'honneur, dont nous avons vu qu'ils sont étroitement imbriqués.

Ces différents exemples nous montrent que l'ensemble des pratiques, quelles qu'elles soient et à quelque niveau qu'elles se situent, prennent en compte la gestion de l'aléa : aussi bien les pratiques agropastorales que les principes de gestion des espaces et l'institution de réseaux d'échange, aussi bien les alliances politiques et matrimoniales que les différents pactes de protection et les liens de partenariat qui aujourd'hui prennent le relais. Elles se complètent entre elles et leurs fonctions se complètent, de sorte qu'il est difficile, voire illusoire, de faire la part des choses entre des faits qui relèveraient de risques "naturels" d'une part, et ceux qui relèveraient de risques «sociaux» d'autre part. La notion même de risque et de "société à risque " pose problème tant la frontière entre risque et normalité semble fragile et toute relative. En ce sens, elle ne semble pas opérante. En revanche, dans le sens où l'ensemble de la société s'organise par rapport aux aléas et dans la mesure où cette dimension particulière structure véritablement le corps social, le concept de risque prend incontestablement toute sa signification. D'une part, toute la construction collective repose sur la gestion des risques, lesquels, il ne faut pas l'oublier, peuvent aller jusqu'à menacer les conditions de reproduction des unités domestiques, donc de la société; dans ce contexte hautement aléatoire, ce n'est, en effet, qu'au prix d'une très forte organisation collective que les activités économiques peuvent se réaliser. D'autre part, ce sont la cohésion et l'identité même des groupes qui puisent leurs racines dans cette dynamique.

Techniques \& Culture, 38 | 2002 


\section{BIBLIOGRAPHIE}

Agabi, C.

1994 « Colactation (pacte de “Tad'a") », pp. 2 046-2 047, in Encyclopédie berbère, fasc. XIII. Aix-enProvence : Edisud.

Amahan, Ali

1998 Mutations sociales dans le Haut Atlas : les Ghoujdama. Paris : Éditions de la Maison des Sciences de l'Homme.

Bédoucha, Geneviève

1987 «L'eau, l'amie du puissant ». Une communauté oasienne du Sud-tunisien. Paris : Éditions des archives contemporaines.

Berque, Jacques

1953 « Notes sur l'histoire des échanges dans le Haut Atlas occidental », Annales E.S.C. 3 : 289-314.

1955 Structures sociales du Haut Atlas. Paris : Presses Universitaires de France.

1978 L'intérieur du Maghreb. XVe-XIXe siècle. Paris : Gallimard.

Berque, Jacques \& J.-P. Charnay (eds)

1967 L'ambivalence dans la culture arabe. Paris : Anthropos.

Bourbouze, Alain

1982 L'élevage dans la montagne marocaine. Organisation de l'espace et utilisation des parcours par les éleveurs du Haut Atlas. Doctorat de l'Institut National Agronomique de Paris.

Bourdieu, Pierre

1972 Esquisse d'une théorie de la pratique, précédé de trois études d'ethnologie kabyle. Genève-Paris : Droz.

Bruno, Henri \& Georges-Henri Bousquet

1946 « Contribution à l'étude des pactes de protection et d'alliance chez les Berbères du Maroc Central », Hespéris XXXIII : 353-371.

Coursimault (Capitaine)

1917 « La 'Ttatta' », Les archives berbère 2 (3) : 261-264.

Couvreur, G.

1968 « La vie pastorale dans le Haut Atlas central », Revue de géographie marocaine 13 : 5-54.

Cresswell, Robert

1970 « Parenté et propriété foncière dans la montagne libanaise », Études rurales 40 : 7-79.

Doutté, Edmond

1914 Missions au Maroc. En tribu. Paris : Paul Geuthner.

Dresch, Jean

1941 Commentaire des cartes sur les genres de vie de montagne dans le Massif central du Grand Atlas. Publications de l'Institut des Hautes Études Marocaines, T. XXXV. Tours : Arrault et Cie.

Garrigues-Cresswell, Martine

1985 « Pasteurs-agriculteurs du Haut Atlas occidental : tribesmen ou peasants? », Production pastorale et société $16: 5-31$. 
1987 « La complémentarité verticale dans le Haut Atlas », Bulletin économique et social du Maroc

159-160-161 : 43-52.

Gellner, Ernest

1969 Saints of the Atlas. Chicago : The University of Chicago Press.

Hart, D.

1981 Dadda 'Atta and his Forty Grandsons. The Socio-Political Organisation of the Ait 'Atta of the Southern Morocco. Cambridge : Middle East and North African Studies Press Ltd.

Jamard, Jean-Luc

1972 «Confréries religieuses et bipartition sociale », pp. 476-488, in Mélanges de la Casa de

Velázquez, Vol. VIII. Paris : Éditions De Boccard.

Jamous, Raymond

1981 Honneur et baraka. Les structures sociales traditionnelles dans le Rif. Paris : Éditions de la Maison des Sciences de l'Homme.

Lecestre-Rollier, Béatrice

1992 Anthropologie d'un espace montagnard. Les Ayt Bou-Guemez du Haut Atlas marocain. Thèse de Doctorat. Paris : Université René-Descartes.

Marcy, Georges

1936 « L'alliance par colactation (tâd'a) chez les berbères du Maroc central », in « Deuxième

Congrès de la Fédération des Sociétés savantes de l'Afrique du nord », Revue africaine 79 : 957-973.

1941 «Les vestiges de la parenté maternelle en droit coutumier berbère et le régime des successions touarègues ", Revue africaine 85 : 187-211.

Montagne, Robert

1930 Les Berbères et le Makhzen dans le Sud du Maroc. Essai sur la transformation politique des Berbères sédentaires (groupe Chleuh). Paris : Alcan.

Murra, John V.

1975 Formaciones económicas y políticas del mundo andino. Lima : Instituto de Estudios Peruanos.

Pascon, Paul

1977 Le Haouz de Marrakech, 2 tomes. Rabat : Éditions marocaines et internationales.

Patai, R.

1962 Golden River to Golden Road. Society, Culture, and Change in the Middle East. Philadelphia :

University of Pennsylvania Press.

Westermarck, Edward

1907 « L' 'âr, or the transference of conditional curses in Morocco », pp. 361-374, in

Anthropological Essays presented to E.B. Taylor. Oxford.

1926 Ritual and Belief in Morocco. London : Macmillan and Co.

1972 Marriage ceremonies in Morocco. London : Curzon Press (1ère édition 1914).

Xanthakou, Margarita

1993 Faute d'épouses, on mange des sœurs. Réalités du célibat et fantasmatique de l'inceste dans le Magne

(Grèce). Paris : Éditions de l'EHESS («Cahiers de l'Homme », 32) 


\section{NOTES}

1. Une version préliminaire de ce texte a été présentée à la table ronde « Risques et idéologie étatique. La construction identitaire des groupes sociaux ou ethniques », organisée par l'UPR 191 les 14 et 15 mai 1998.

2. Nous avons respecté l'orthographe des termes berbères et arabes tels qu'ils apparaissent dans les citations. La très grande variabilité des parlers berbères explique sans doute pour une part les différences de transcription d'un auteur à l'autre.

3. D'origine américaine, le maïs est attesté dans le Haut Atlas depuis le XVIIIe siècle (Berque 1978). Il a rapidement pris une place importante dans les assolements.

4. John Murra (1975) a été l'un des premiers à proposer le concept de complémentarité verticale dans ses analyses des régions andines. Au Maroc, parmi les précurseurs, citons les travaux de Jean Dresch (1941), de Jacques Berque (1953 et 1955) et de de Paul Pascon (1977), qui ont beaucoup apporté à la compréhension des systèmes d'échange entre économies complémentaires.

5. Sans entrer dans le détail des fondements de la propriété collective, précisons simplement que chaque village, ou groupe de villages, possède ses propres parcours pastoraux dont l'accès est réservé aux membres de la communauté villageoise. 6. L'exploitation forestière est officiellement du ressort de l'État depuis que les fondements de la domanialité furent posés au Maroc, dès les débuts du protectorat français. Cependant, les populations montagnardes refu-sèrent de se voir dépossédées de leurs droits sur les espaces boisés. Aussi le code forestier ne fut-il jamais véritablement appliqué dans les zones reculées du royaume et aujourd'hui encore, bien que la situation évolue, le droit coutumier forestier promulgué par les assemblées villageoises, responsables des espaces collectifs, perdure dans bon nombre de hautes vallées.

7. Avec la diminution du nombre et de la taille des troupeaux surtout, certaines routes de la transhumance sont abandonnées ou plutôt, de nouvelles pratiques pastorales apparaissent : l'utilisation du camion pour déplacer les animaux, le placement des bêtes en hivernage chez des éleveurs du Sud ou du Nord, l'achat de complément alimentaire qui permet au troupeau, s'il n'est pas trop important, de demeurer dans la vallée pendant la mauvaise saison, etc. Autrefois importante par son ampleur et le nombre d'animaux concernés - Couvreur (1968) évoque, pour les ayt Bou-Guemez, un total de plus d'une dizaine de milliers de bêtes et les témoignages des éleveurs permettent de penser que l'ensemble des troupeaux de la vallée était jadis concerné-, la transhumance hivernale n'est plus entreprise aujourd'hui que par les plus importants troupeaux.

8. Les caprins se déplacent plus rapidement et plus agilement que les ovins en terrain escarpé; ils ont un comportement alimentaire plus varié, bien adapté au couvert ligneux; en revanche, ils craignent davantage le froid et l'humidité des hauts parcours. 9. Le bur désigne au Maroc le terroir non irrigué où se pratique une agriculture sèche.

10. Signalons la controverse entre Berque et Montagne au sujet de l'existence des leff-s en montagne.

11. Chez les ayt Atta, le pacte de colactation est nommé tafergant, selon Hart (1981).

12. Les lignages chorfa affirment descendre du prophète; les lignages maraboutiques se rattachent à un saint vénéré localement.

13. On la trouve aussi dans diverses sociétés du pourtour méditerranéen : en Grèce traditionnelle, par exemple, elles est connue sous le nom de 'soghambros, littéralement 
"gendre [à] l'intérieur » (ou " gendre à domicile »), là où existaient encore il y a peu des patrilignages (Xanthakou 1993).

14. Plusieurs auteurs ont souligné, dans la culture arabe, l'ambivalence lexicale qui transparaît notamment dans le phénomène des ad'däd, mots possédant deux significations apparemment opposées (Berque et Charnay 1967).

\section{RÉSUMÉS}

Les sociétés du Haut Atlas marocain s'organisent autour de la gestion des aléas, qu'il s'agisse d'aléas d'ordre climatique ou bien d'aléas d'ordre socio-politique. Elles y répondent par différentes formes de complémentarité verticale et par une grande souplesse dans les stratégies pastorales. Elles multiplient aussi les pactes d'alliance et de protection qui s'expriment dans le langage de la parenté. L'accent est mis sur l'actualité de ces pratiques.

Managing random events. The societies of the Moroccan High Atlas

Moroccan High Atlas societies are organized so as to manage random events, whether these latter consist of ecological phenomena or socio-political behavior. The responses represent different forms of vertical integration and show remarkable powers of adjustments in pastoral strategies. They also take the form of pacts of alliance and protection, as expressed in kinship language. The article underlines that these practices are up-to-date.

\section{Gestión de la incertidumbre. La sociedades del Alto Atlas marroquí}

La sociedades del Alto Atlas marroquí se organizan alrededor de la gestión de la incertidumbre, sea esta de orden climático o socio-político. De un lado, se enfrentan a ella a través de distintas formas de complementariedad vertical y de una gran flexibilidad de las estrategias de pastoreo. De otro lado, multiplican los pactos de alianza y de protección que se expresan en el idioma del parentesco. Para acabar, las autoras hacen énfasis en la vigencia que estas prácticas tienen en la actualidad.

\section{INDEX}

Mots-clés : Maroc, Agro-pastoralisme, aléas, complémentarité verticale, montagnes, parenté, pactes d'alliance, pactes de protection

\section{AUTEURS}

\section{MARTINE GARRIGUES-CRESSWELL}

Université Paris V, 12 rue Cujas, 75005 Paris; membre de « Genèse et Transformation des Mondes Sociaux », FRE 2324, CNRS.

\section{BÉATRICE LECESTRE-ROLLIER}

Université de Caen; membre de «Techniques et culture », UMR 8098, CNRS, 27 rue Paul Bert, 94204 Ivry Cedex. 